\title{
Dromion solutions for nonlinear electron acoustic waves in space plasmas
}

\author{
S. S. Ghosh ${ }^{1}$, A. Sen ${ }^{2}$, and G. S. Lakhina ${ }^{1}$ \\ ${ }^{1}$ Indian Institute of Geomagnetism, Dr. Nanabhai Moos Marg, Colaba, Mumbai, 400 005, India \\ ${ }^{2}$ Institute for Plasma Research, Bhat, Gandhinagar, 382 428, India
}

Received: 24 January 2002 - Revised: 12 June 2002 - Accepted: 13 June 2002

\begin{abstract}
Recent high resolution measurements of POLAR and FAST satellites have revealed two-dimensional coherent structures in the polar cap boundary layer (PCBL) region. Since the ion temperature is often observed to be greater than the electron temperature in this region, electron acoustic waves can exist as a normal mode of the plasma system. It is shown that the nonlinear evolution of such waves can be modelled by the coupled Davey-Stewartson I equations. These equations, which are a generalization of the nonlinear Schrödinger equation to two dimensions, admit exponentially localized solutions called dromions. A detailed parametric characterization of the regions of existence of such solutions is presented in the context of the PCBL region.
\end{abstract}

\section{Introduction}

Solitons, which are exponentially localized wave forms in $(1+1)$ dimension, are well-known as exact solutions of certain integrable partial differential equations. In the past few decades they have been extensively studied and applied in a variety of fields such as physics, chemistry, biology, neurophysics, etc. They have also figured prominently in the interpretation of several wave structure observations in space plasmas. However, work related to two- and threedimensional generalizations of solitons is of more recent origin and consequently is relatively less well-known. One of the earliest work is that of Kadomtsev and Petviashvili (1970), who constructed a two-dimensional generalization of the K-dV equation. Their model equation, known as the Kadomtsev-Petviashvili (KP) equation, admits a variety of nonlinear solutions. Line solitons are the simplest kind and are exponentially localized in a single direction (say $x$ ) and propagate in the $(x, y)$ plane. Their equation also admits lump or algebraic solitons which decay polynomially (i.e. as $\left.O\left(1 / x^{2}, 1 / y^{2}\right)\right)$ in all directions (Satsuma and Ablowitz, 1979). A host of other independent equations governing sim-

Correspondence to: S. S. Ghosh (sukti@iig.iigm.res.in) ilar two-dimensional solitary structures have been studied by many authors (Djordjevic and Redekopp, 1977; Manakov, 1976; Ablowitz and Segur, 1979). However, none of these studies dealt with a nonlinear solution that had an exponential decay in both dimensions. This lacuna was removed by the work of Boiti et al. (1989), who found coherent localized structures as a solution of $(2+1)$ dimensional integrable partial differential equations which, unlike a lump or algebraic solitons (Satsuma and Ablowitz, 1979; Janaki et al., 1991), decayed exponentially in both directions in the ( $x$, $y$ ) plane. One characteristic feature of these solutions is that they involve two field variables. One of the variables represents the physical entity which is being evolved (the electric field amplitude, for example) and displays a localized structure in space. The other auxiliary variable shows a plane wave character with vanishing physical fields at the boundaries and is often called a ghost soliton (Hietarinta, 1990). The dromion solutions also require special time dependent boundary conditions which are described by those ghost solitons. These ghost solitons are in fact the line solitons (Fokas and Santini, 1989) mentioned earlier and represent the "tracks" (dromos in Greek), along which the localized lumps of the physical variable travel. In fact, the lumps are located precisely at the intersection of two such tracks of the auxiliary variable. Since they are "driven" by their boundaries, they were, therefore, named as dromions (Fokas and Santini, 1990). Ever since their discovery the mathematical properties of dromions have been investigated by many authors both analytically (Santini, 1990; Hietarinta, 1990; Hietarinta and Hirota, 1990; Radha and Lakshmanan, 1995), as well as numerically (Besse and Bruneau, 1998; Nishinari and Yajima, 1994; Nishinari et al., 1996).

A large class of two-dimensional partial differential equations have been shown to possess dromion solutions (Radha and Lakhsmanan, 1997b; Guil and Manas, 1996; Imai, 1997; Ruan and Chen, 1999; Lou and Ruan, 2001). One of the most well-known of such equations is the so-called DaveyStewartson-I equation (DS-I) (Davey and Stewartson, 1974), which is a two-dimensional generalization of the nonlinear 
Schrödinger equation (NLSE). While the NLSE finds extensive applications in plasma physics, including the modelling of several wave phenomena in space plasmas, the DSI equations have received scant attention from plasma and space scientists. A rare exception is the work of Nishinari et al. (1994), who have shown, with the help of a suitable reductive perturbation method, that the nonlinear evolution of an ion acoustic wave travelling in a magnetized plasma can be modelled by a DS-I type of equation. Similar considerations might, therefore, apply for other modes in a plasma, particularly when their nonlinear stationary states display two- or three-dimensional structures. Our present work is motivated by such a consideration particularly in the context of space plasma observations which provide a rich source of such potential structures. For instance, the GEOTAIL spacecraft was one of the earliest (Matsumoto et al., 1994a) to detect interesting waveforms consisting of a series of solitary pulses that give rise to the broad band electrostatic noise (BEN) (Scarf et al., 1974; Gurnet et al., 1976) in the plasma sheet boundary layer. These structures were commonly called ESW, i.e. Electrostatic Solitary Waves. However, it was soon noticed that the ESWs do not exhibit the characteristics of solitons or envelope solitons (Matsumoto et al., 1994b). It was further observed that their evolution depended crucially on the ion temperature and electron flow (Kojima et al., 1994). This led Omura et al. (1994) to propose a 1-D BGK mode model to explain these spiky structures. They argued that the BEN/ESW structures can be sustained when the condition $T_{\mathrm{i}}>T_{\mathrm{e}}$ is satisfied (Omura et al., 1994). Further credence to this model was provided by experimental observations of energetic ions in the magnetospheric tail and ring currents (Baumjohann et al., 1989; Buti et al., 1980). However, recent high resolution measurements from POLAR and FAST satellites have revealed 2-D structures for BEN (Franz et al., 1998) which cannot be explained by the previously proposed one-dimensional models (Omura et al., 1996). The observed two-dimensional electrostatic structures consist of both monopolar and bipolar pulses and occur in a region where typically $T_{\mathrm{i}}>T_{\mathrm{e}}$ (Tsurutani et al., 1998). Past theoretical works have pointed out the possibility of exciting linear electron acoustic waves in such a regime (Lashmore-Davies and Martin, 1973) and also discussed their one-dimensional nonlinear properties (Buti, 1980; Bharuthram and Shukla, 1988). There is also reasonable experimental evidence (gleaned from detailed analysis of FAST and Viking data) of the presence of electron acoustic waves associated with BEN activity in auroral plasmas (Pottelette et al., 2001; Dubuloz et al., 1991b). Comparative studies of Singh and Lakhina (2001); Singh et al. (2001) and Dubuloz et al. (1991a) further highlight the consistency between the theoretical models and spacecraft data for both linear and nonlinear regimes. It thus becomes important to examine the nonlinear evolution of these waves and to look for possible two-dimensional saturated stationary structures. We have carried out such an investigation in this paper and have shown that the nonlinear evolution of the electron acoustic wave can be modelled by the system of Davey-Stewartson-I equations under certain conditions. Further, we have used these conditions to delineate the parametric regions for the existence of dromion solutions for two-dimensional electron acoustic waves.

The paper is organized as follows. In the next section (Sect. 2) we derive a general nonlinear evolution equation for finite amplitude electron acoustic waves which are travelling in an arbitrary direction. The derivation is based on the reductive perturbation method as applied to a two-fluid plasma model. The special case of pure perpendicular wave propagation is considered in Sect. 3 and the conditions necessary for the evolution equations to reduce to the DS-I equations are stated. These conditions are used to numerically delineate the parametric regimes for the occurrence of dromion solutions. An analytic form for the dromion solution using the Hirota bilinear method is discussed as a special case in Appendix B. Section 4 discusses the conditions necessary for the general evolution equation to reduce to the DS-I equations. These conditions are further numerically analyzed. In Sect. 5 some comparisons are made with the actual experimental observations in space and Sect. 6 provides a brief summary and concluding remarks.

\section{Derivation of the nonlinear evolution equations}

For $T_{i}>T_{e}$, the electron acoustic wave is a normal mode of a magnetized plasma with a typical phase velocity of $\sqrt{\left(T_{i} / m_{e}\right)}$. This mode is analogous to the ion-acoustic wave with the roles of electrons and ions reversed, that is, the electron mass now provides the inertial restoring force to balance the ion thermal pressure force. An appropriate fluid description of this mode is provided by considering the electron equations of continuity and motion and assuming the ions to obey a Boltzmann distribution. The Boltzmann description for ions is justified on account of their large thermal energy which allows one to treat them as an unmagnetized species. We adopt the following normalized set of equations (Ghosh et al., 1996),

$$
\begin{aligned}
& \frac{\partial n}{\partial t}+\nabla \cdot(n \boldsymbol{v})=0, \\
& \frac{\partial \boldsymbol{v}}{\partial t}+(\boldsymbol{v} \cdot \nabla) \boldsymbol{v}+3 \beta n \nabla n-\nabla \phi+\alpha(\boldsymbol{v} \times \boldsymbol{b})=0, \\
& \nabla^{2} \phi=n_{\mathrm{e}}-n_{\mathrm{i}}=n_{\mathrm{e}}-\exp (-\phi),
\end{aligned}
$$

where $\alpha\left(=\omega_{\mathrm{ce}} / \omega_{\mathrm{pe}}\right)$ is the ratio of electron cyclotron and electron plasma frequencies, $\beta\left(=T_{\mathrm{e}} / T_{\mathrm{i}}\right)$ is the electron to ion temperature ratio, $\boldsymbol{b}=(0,0,1)$ is normalized by the ambient magnetic field $B_{0}$ and $\phi\left(=e\left|\phi^{\prime}\right| / T_{i}\right)$ is the normalized electrostatic potential, with $\phi^{\prime}$ being the actual measured potential. All the space variables are normalized by the ion Debye length $\left(\lambda_{\mathrm{i}}\right)$, time by reciprocal of electron plasma frequency $\left(\omega_{\mathrm{pe}}^{-1}\right)$, velocities by electron acoustic speed $\left(c_{\mathrm{s}}=\sqrt{T_{\mathrm{i}} / m_{\mathrm{e}}}\right)$ and number densities by the ambient plasma density $n_{0}$. We now assume a perturbation of the 
form $\sim \exp [\imath(\boldsymbol{k} \cdot \boldsymbol{r}-\omega t)]$, where $\boldsymbol{k}=\left(0, k_{\perp}, k_{\|}\right)$is the wave vector and we adopthe reductive perturbation expansion technique (Nishinari et al., 1994) to derive the nonlinear evolution equation of the perturbation. We expand the physical quantities as

$n=1+\sum_{\mathrm{n}=1}^{\infty} \epsilon^{\mathrm{n}} \sum_{1=-\infty}^{\infty} n_{1}^{(\mathrm{n})} \exp [\imath l(\boldsymbol{k} \cdot \boldsymbol{r}-\omega t)]$,

$\phi=\sum_{\mathrm{n}=1}^{\infty} \epsilon^{\mathrm{n}} \sum_{\mathrm{l}=-\infty}^{\infty} \phi_{1}^{(\mathrm{n})} \exp [\mathrm{l} l(\boldsymbol{k} \cdot \boldsymbol{r}-\omega t)]$,

$\boldsymbol{v}=\sum_{\mathrm{n}=1}^{\infty} \epsilon^{\mathrm{n}} \sum_{\mathrm{l}=-\infty}^{\infty}\left(\begin{array}{c}v_{\mathrm{xl}}^{(\mathrm{n})} \\ v_{\mathrm{yl}}^{(\mathrm{n})} \\ v_{\mathrm{zl}}^{(\mathrm{n})}\end{array}\right) \exp [l l(\boldsymbol{k} \cdot \boldsymbol{r}-\omega t)]$.

We further introduce the following stretched variables (Nishinari et al., 1994)

$$
\begin{array}{llrl}
\xi & =\epsilon x, & \eta & =\epsilon\left(y-M_{\mathrm{y}} t\right) \\
\zeta=\epsilon\left(z-M_{\mathrm{z}} t\right), & \tau & =\epsilon^{2} t,
\end{array}
$$

where $M_{\mathrm{y}}, M_{\mathrm{z}}$ are the respective group velocities in the perpendicular and parallel directions. The choice of the stretched variables and the consequent scaling of the various terms is guided primarily by the nature of dispersion of the linear waves, as has been extensively discussed in the literature (Lokenath, 1997). Transforming all independent variables by Eq. (7), we expand Eqs. (1-3) by using Eqs. (4-6) and carry out a systematic balancing of terms at each order of $\epsilon$.
To the first order in $\epsilon$ we obtain

$$
\begin{aligned}
& \epsilon: l=1 ; \quad \phi_{1}^{(1)}=-K_{1} n_{1}^{(1)} \text {, } \\
& v_{\mathrm{x} 1}^{(1)}=-l a_{2} k_{\perp} K_{2} n_{1}^{(1)} \text {, } \\
& v_{\mathrm{y} 1}^{(1)}=a_{1} k_{\perp} K_{2} n_{1}^{(1)} \text {, } \\
& v_{\mathrm{z} 1}^{(1)}=\frac{k_{\|}}{\omega} K_{2} n_{1}^{(1)} \text {, }
\end{aligned}
$$

where, for convenience, the coefficients $K_{\mathrm{i}} \mathrm{s}, a_{\mathrm{i}} \mathrm{s}(i, j=$ $1,2,3$, etc.) and those occurring in subsequent expressions below are listed in Appendix A. Combining the above expressions leads to the linear dispersion relation for the electron acoustic wave in a warm electron fluid, namely

$$
\omega^{4}-\omega^{2}\left(\alpha^{2}+|k|^{2} K_{2}\right)+\alpha^{2} k_{\|}^{2} K_{2}=0 .
$$

For an unmagnetized plasma $(\alpha=0)$ it reduces to the usual linear electron acoustic dispersion

$\frac{\omega}{k}=\sqrt{K_{2}}$,

while for a strong magnetic field and perpendicular propagation it reduces to

$\frac{\omega}{k}=\sqrt{\frac{\alpha^{2}}{k^{2}}+K_{2}}$,

indicating a stronger dispersion which is consistent with our choice of scaling (Lokenath, 1997).

The next order balance yields

$$
\begin{aligned}
\epsilon^{2}: l=0 ; \quad \phi_{0}^{(2)} & =-\left(n_{0}^{(2)}-K_{1}^{2}\left|n_{1}^{(1)}\right|^{2}\right), \\
v_{\mathrm{x} 0}^{(2)} & =0, \\
v_{\mathrm{y} 0}^{(2)} & =-2 c_{2}\left|n_{1}^{(1)}\right|^{2}, \\
\epsilon^{2}: l=1 ; \quad \phi_{1}^{(2)} & =-K_{1}\left[n_{1}^{(2)}+2 \imath K_{1}\left(\boldsymbol{k} \cdot \nabla_{\xi}\right) n_{1}^{(1)}\right], \\
v_{\mathrm{x} 1}^{(2)} & =-\imath K_{2}\left(a_{2} k_{\perp} n_{1}^{(2)}+a_{1} \frac{\partial n_{1}^{(1)}}{\partial \xi}\right)+2 a_{2} k_{\perp}\left(X_{\mathrm{x} \eta} \frac{\partial n_{1}^{(1)}}{\partial \eta}+X_{\mathrm{x} \zeta} \frac{\partial n_{1}^{(1)}}{\partial \zeta}\right), \\
v_{\mathrm{y} 1}^{(2)} & =K_{2}\left(a_{1} k_{\perp} n_{1}^{(2)}+a_{2} \frac{\partial n_{!}^{(1)}}{\partial \xi}\right)+\imath a_{1} k_{\perp}\left(X_{\mathrm{y} \eta} \frac{\partial n_{1}^{(1)}}{\partial \eta}+X_{\mathrm{y} \zeta} \frac{\partial n_{1}^{(1)}}{\partial \zeta}\right), \\
v_{\mathrm{z} 1}^{(2)} & =\frac{k_{\|}}{\omega}\left[K_{2} n_{1}^{(2)}+\imath\left(X_{\mathrm{z} \eta} \frac{\partial n_{1}^{(1)}}{\partial \eta}+X_{\mathrm{z} \zeta} \frac{\partial n_{1}^{(1)}}{\partial \zeta}\right)\right], \\
\phi_{2}^{(2)} & =-\kappa_{1}\left\{S_{\mathrm{n}}-\frac{1}{2} K_{1}^{2}\right\}\left(n_{1}^{(1)}\right)^{2}, \\
\epsilon^{2}: l=2 ; \quad n_{2}^{(2)} & =S_{\mathrm{n}}\left(n_{1}^{(1)}\right)^{2}, \\
&
\end{aligned}
$$




$$
\begin{aligned}
& v_{\mathrm{x} 2}^{(2)}=-\imath k_{\perp} S_{\mathrm{x}}\left(n_{1}^{(1)}\right)^{2}, \\
& v_{\mathrm{y} 2}^{(2)}=k_{\perp} S_{\mathrm{y}}\left(n_{1}^{(1)}\right)^{2}, \\
& v_{\mathrm{z} 2}^{(2)}=k_{\|} S_{\mathrm{z}}\left(n_{1}^{(1)}\right)^{2} .
\end{aligned}
$$

The above relations from the second order balance give us the relations for the group velocities as

$$
\begin{aligned}
& M_{\mathrm{y}}=2 k_{\perp} \frac{a_{1} K_{2}-c_{1} K_{1}^{2}}{1+c_{3} K_{2}}, \\
& M_{\mathrm{z}}=2 k_{\|} \frac{\frac{K_{2}}{\omega}-c_{1} K_{1}^{2}}{1+c_{3} K_{2}} .
\end{aligned}
$$

Finally, in the third order we obtain

$\epsilon^{3}: l=1 ; \quad \phi_{1}^{(3)}=-K_{1}\left[n_{1}^{(3)}+K_{1}\left\{\nabla_{\xi}^{2} n_{1}^{(1)}+2 \imath\left(\boldsymbol{k} \cdot \nabla_{\xi}\right) n_{1}^{(2)}-n_{1}^{(1)} n_{0}^{(2)}\right\}-4 K_{1}^{2}\left(\boldsymbol{k} \cdot \nabla_{\xi}\right)^{2} n_{1}^{(1)}+K_{1}^{3} n_{1}^{(1)}\left|n_{1}^{(1)}\right|^{2}\right]$

Using Eqs. (8)-(19) in Eqs. (1)-(3) and solving for the third order of $\epsilon$ we obtain the following set of coupled equations

$$
\begin{aligned}
& -M_{\mathrm{y}}^{2} \frac{\partial^{2} n_{0}^{(2)}}{\partial \eta^{2}}-2 M_{\mathrm{y}} M_{\mathrm{z}} \frac{\partial^{2} n_{0}^{(2)}}{\partial \eta \partial \zeta}+\left(3 \beta+1-M_{\mathrm{z}}^{2}\right) \frac{\partial^{2} n_{0}^{(2)}}{\partial \zeta^{2}}+p_{\eta \eta} \frac{\partial^{2}\left|n_{1}^{(1)}\right|^{2}}{\partial \eta^{2}}+p_{\eta \zeta} \frac{\partial^{2}\left|n_{1}^{(1)}\right|^{2}}{\partial \eta \partial \zeta}+p_{\zeta \zeta} \frac{\partial^{2}\left|n_{1}^{(1)}\right|^{2}}{\partial \zeta^{2}}=0 \\
& -M_{\mathrm{y}}^{2} \frac{\partial^{2} v_{\mathrm{z} 0}^{(2)}}{\partial \eta^{2}}-2 M_{\mathrm{y}} M_{\mathrm{z}} \frac{\partial^{2} v_{\mathrm{z} 0}^{(2)}}{\partial \eta \partial \zeta}+\left(3 \beta+1-M_{\mathrm{z}}^{2}\right) \frac{\partial^{2} v_{\mathrm{z} 0}^{(2)}}{\partial \zeta^{2}}+q_{\eta \eta} \frac{\partial^{2}\left|n_{1}^{(1)}\right|^{2}}{\partial \eta^{2}}+q_{\eta \zeta} \frac{\partial^{2}\left|n_{1}^{(1)}\right|^{2}}{\partial \eta \partial \zeta}+q_{\zeta \zeta} \frac{\partial^{2}\left|n_{1}^{(1)}\right|^{2}}{\partial \zeta^{2}}=0
\end{aligned}
$$

for $l=0$ and

$\iota \frac{\partial n_{1}^{(1)}}{\partial \tau}+\mu_{\xi \xi} \frac{\partial^{2} n_{1}^{(1)}}{\partial \xi^{2}}+\mu_{\eta \eta} \frac{\partial^{2} n_{1}^{(1)}}{\partial \eta^{2}}+\mu_{\eta \zeta} \frac{\partial^{2} n_{1}^{(1)}}{\partial \eta \partial \zeta}+\mu_{\zeta \zeta} \frac{\partial^{2} n_{1}^{(1)}}{\partial \zeta^{2}}-\left(\mu_{02} n_{0}^{(2)}+k_{\|} v_{\mathrm{z} 0}^{(2)}\right) n_{1}(1)-\mu_{11}\left|n_{1}^{(1)}\right|^{2} n_{1}^{(1)}=0$

for $l=1$, where all the coefficients are given in Appendix A. Equations (20)-(22) provide a generalized description of the evolution of the nonlinear wave packet in three dimensions. They can be further simplified by assuming special situations which we discuss in the subsequent sections.

\section{Perpendicular propagation of the wave}

For pure perpendicular propagation of the wave we can set $k_{\|}=M_{\mathrm{z}}=0$. We can further assume $\partial_{\xi} \rightarrow 0$. Due to the symmetry of the system, all the cross derivative terms then vanish. Substituting these conditions, Eqs. (22) and (20), respectively, reduce to

$$
\begin{aligned}
& l \frac{\partial n_{1}^{(1)}}{\partial \tau}+\mu_{\eta \eta} \frac{\partial^{2} n_{1}^{(1)}}{\partial \eta^{2}}+\mu_{\zeta \zeta} \frac{\partial^{2} n_{1}^{(1)}}{\partial \zeta^{2}} \\
& \quad-\left(\mu_{02} n_{0}^{(2)}+\mu_{11}\left|n_{1}^{(1)}\right|^{2}\right) n_{1}^{(1)}=0, \\
& -M_{\mathrm{y}}^{2} \frac{\partial^{2} n_{0}^{(2)}}{\partial \eta^{2}}+(3 \beta+1) \frac{\partial^{2} n_{0}^{(2)}}{\partial \zeta^{2}}+K_{4} \frac{\partial^{2}\left|n_{1}^{(1)}\right|^{2}}{\partial \eta^{2}}=0 .
\end{aligned}
$$

Substituting

$n_{1}^{(1)}=A ; n_{0}^{(2)}=Q$,

and

$\mu_{\eta \eta}=d_{\eta} ; \mu_{\zeta \zeta}=d_{\zeta}$
Eqs. (23) and (24) can be rewritten as

$$
\begin{aligned}
& \iota A_{\tau}+d_{\eta} A_{\eta \eta}+d_{\zeta} A_{\zeta \zeta}-\left(\mu_{02} Q+\mu_{11}|A|^{2}\right) A=0, \\
& -M_{\mathrm{y}}^{2} Q_{\eta \eta}+(3 \beta+1) Q_{\zeta \zeta}+K_{4}|A|_{\zeta \zeta}^{2}=0,
\end{aligned}
$$

where the subscripts of the dependent variables $A$ and $Q$ denote partial derivatives. The two coupled Eqs. (25) and (26) have the form of the DS-I equations, provided the condition

$d_{\eta} / d_{\zeta}>0 ; \mu_{11}>0$

is satisfied, with $\mu_{02}$ being always positive. Condition (27) provides us with the necessary criterion to explore the physical parameter space where dromion structures can appear as nonlinear saturated states of the electron acoustic wave. We have carried out this exploration numerically and Figs. 1a-c represent the parameter space at different values of $\beta$ (e.g. $\beta=0,0.1$ and 1 , respectively) for which the condition (27) is satisfied. In these parameter regions, the nonlinear evolution for electron acoustic waves is governed by the DS-I equation and hence may admit dromion or breather type solutions. For some special values of the coefficients, the coupled equations (viz. Eqs. 25 and 26) become analytically integrable and thus, when solved for appropriate initial-boundary conditions, give dromions. One such technique is the Hirota bilinear method, as discussed, for example in Radha and Lakshmanan (1997a); Radha and Lakhsmanan (1997b). We have illustrated this method in Appendix B and a typical form of 




Fig. 1a. Existence domain for DS-I equation ; $\beta=0$.

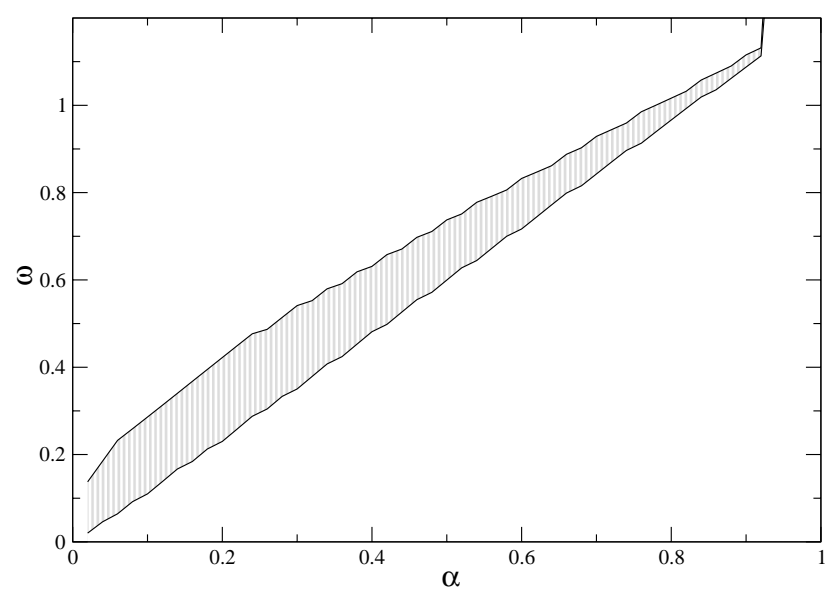

Fig. 1b. Existence domain for DS-I equation ; $\beta=0.1$.

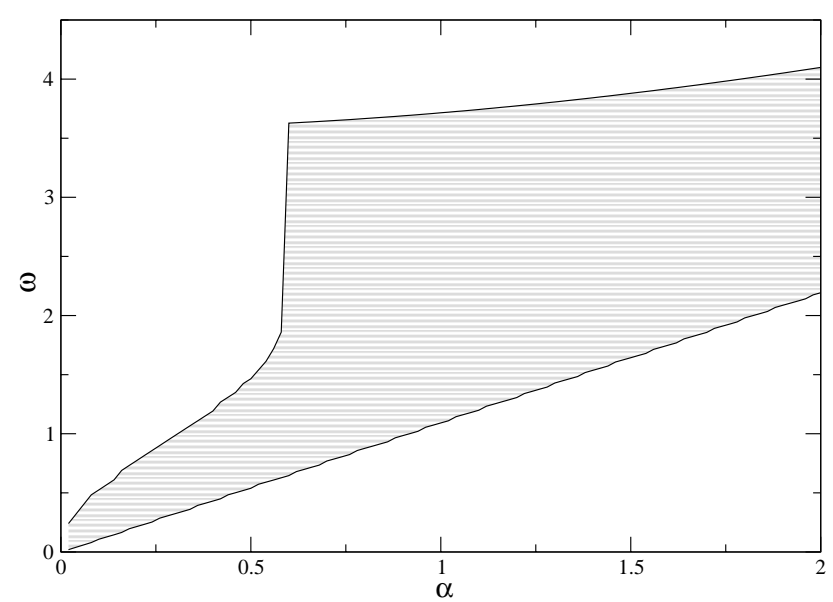

Fig. 1c. Existence domain for DS-I equation ; $\beta=1$.

the solution is obtained (Eq. B19). Using an appropriate realistic set of parameters $(\alpha=0.9, \omega=1.098$ from Fig. 1b) which allows the analytic solution for DS-I equations (see Appendix B) and putting them into Eq. (B19), we have obtained an actual analytical solution for dromion, as shown in
Fig. 2. For convenience, the shape of the dromion is assumed to be spherical (i.e. equal width ratio) while the time is taken to be $t=0$. However, the analytic method is restricted only to very specific sets of parameters and for a more general case, one needs to solve the DS-I equations numerically.

In the present paper our primary aim has been to delineate the parametric regions where the evolution equations take the form of the DS-I equations and to further demonstrate from available experimental data that these parametric regimes are physically relevant in the PCBL region. At the moment we note from Figs. 1a-c that the area of the parametric region where condition (27) is satisfied is larger for larger values of $\beta$ (i.e. it increases as $\beta \rightarrow 1$ ). Since space plasma observations show an appreciably large $T_{\mathrm{e}}$ for the $T_{\mathrm{i}} \geq T_{\mathrm{e}}$ (Gage and Reid, 1981), our analysis seems to support the possibilities of finding dromion solutions for those regimes. It is to be noted that for the case of $\beta \sim 1$, the electron acoustic wave tends to become damped due to Landau damping in a pure protonelectron (single species) plasma (Lashmore-Davies and Martin, 1973). However, since the electron component of the auroral plasma often displays a two-temperature character (i.e. a hot and a cold electron species), the electron acoustic wave may still survive in that region without any appreciable damping. Our fluid model treatment is strictly valid when such kinetic effects can be ignored, e.g. in the region of small $\beta$ (say, $\beta_{\max } \approx 0.1$ ), where electron temperatures are not too large. Our further analysis is primarily restricted to such regimes.

\section{Arbitrary propagation}

We now discuss the case of arbitrary propagation, i.e. the case when $k_{\|} \neq 0$. Combining Eqs. (20)-(22) we obtain

$$
\begin{aligned}
& -M_{\mathrm{y}}^{2} \frac{\partial^{2} Q^{\prime}}{\partial \eta^{2}}-2 M_{\mathrm{y}} M_{\mathrm{z}} \frac{\partial^{2} Q^{\prime}}{\partial \eta \partial \zeta}+\left(3 \beta+1-M_{\mathrm{z}}^{2}\right) \frac{\partial^{2} Q^{\prime}}{\partial \zeta^{2}} \\
& +r_{\eta \eta} \frac{\partial^{2}|A|^{2}}{\partial \eta^{2}}+r_{\eta \zeta} \frac{\partial^{2}|A|^{2}}{\partial \eta \partial \zeta}+r_{\zeta \zeta} \frac{\partial^{2}|A|^{2}}{\partial \zeta^{2}}=0, \\
& \iota \frac{\partial A}{\partial \tau}+\mu_{\eta \eta} \frac{\partial^{2} A}{\partial \eta^{2}}+\mu_{\eta \zeta} \frac{\partial^{2} A}{\partial \eta \partial \zeta}+\mu_{\zeta \zeta} \frac{\partial^{2} A}{\partial \zeta^{2}} \\
& \quad-\mu_{02} Q^{\prime} A-\mu_{11}|A|^{2} A=0,
\end{aligned}
$$

where

$Q^{\prime}=n_{0}^{(2)}+\frac{k_{\|}}{\mu_{02}} v_{z 0}^{(2)} ; r_{\alpha \beta}=p_{\alpha \beta}+\frac{k_{\|}}{\mu_{02}} q_{\alpha \beta}$.

The conditions under which the above equations can reduce to the DS-I equations are not as simple as was the case for the perpendicular propagation limit. In addition, in order to satisfy a criterion analogous to Eq. (27), we will also need to ensure that all three cross derivative terms of Eqs. (28) and (29) vanish simultaneously. To derive these conditions it is convenient to adopt the following linear transformation of the independent variables

$\partial_{\eta}=p_{\mathrm{y}} \partial_{\mathrm{y}}+p_{\mathrm{z}} \partial_{\mathrm{z}} ; \quad \partial_{\zeta}=q_{\mathrm{y}} \partial_{\mathrm{y}}+q_{\mathrm{z}} \partial_{\mathrm{z}}$, 


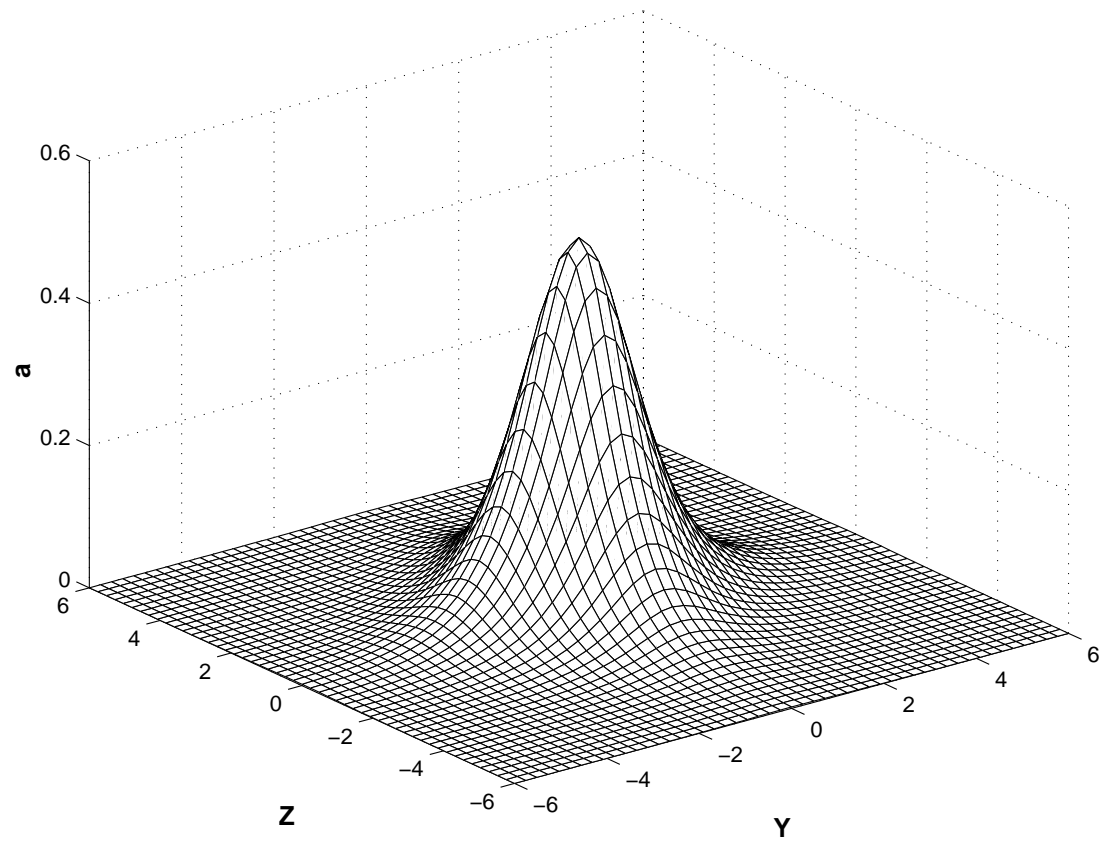

Fig. 2. Analytic solution for a dromion.

where $p_{\mathrm{y}}, p_{\mathrm{z}}, q_{\mathrm{y}}$ and $q_{\mathrm{z}}$ are real numbers. The condition for all the terms containing $\partial_{\mathrm{y}} \partial_{\mathrm{z}}$ to be simultaneously zero is given by

$$
\hat{\boldsymbol{M}} \cdot \hat{\boldsymbol{T}}=0,
$$

where $\hat{\boldsymbol{M}}$ is a $3 \times 3$ matrix consisting of the coefficients of Eqs. (28) and (29) and $\hat{\boldsymbol{T}}$ is a three-component column vector consisting of the transform coefficients, viz. $p_{\mathrm{y}, \mathrm{z}}, q_{\mathrm{y}, \mathrm{z}}$, and are given in Appendix A. Equation (30) can have nontrivial solutions if and only if $\operatorname{det}|\hat{\boldsymbol{M}}|=0$, which leads to the condition

$\mu_{\eta \eta}+\mu_{\zeta \zeta}\left(e_{1}-e_{2} e_{3}\right)-e_{3} \mu_{\eta \zeta}=0$.

The elliptic-hyperbolic nature of the Eqs. (28) and (29) is ensured by

$$
\begin{array}{r}
\mu_{\eta \eta} \mu_{\zeta \zeta}\left(1+E^{2} t^{4}\right)+\mu_{\eta \zeta}\left(\mu_{\eta \eta}+\mu_{\zeta \zeta} E\right)\left(E T^{3}+t\right)+\left(\mu_{\eta \eta}^{2}+\mu_{\eta \zeta}^{2} E+\mu_{\zeta \zeta}^{2} E^{2}\right) t^{2}>0, \\
e_{1}\left(1+E^{2} t^{4}\right)+e_{2}\left(E-e_{1}\right)\left(E T^{3}+t\right)-\left(e_{1}^{2}+e_{2}^{2} E+E^{2}\right) t^{2}>0,
\end{array}
$$

where $t=p_{\mathrm{z}} / q_{\mathrm{z}}$ and $E=e_{1}-e_{2} e_{3}$ and the interrelations among the transformation coefficients, viz. $p_{\mathrm{y}, \mathrm{z}}, q_{\mathrm{y}, \mathrm{z}}$, have been taken into account. Provided Eq. (31) is satisfied, $t$ can be determined from the following second order equation (see Appendix A)

$E t^{2}+2 e_{3} t+1=0$.

Equations (31) to (34), together with the relation $\mu_{11}>0$, complete the set of conditions necessary for ensuring that the generalized (oblique) propagation equations reduce to the DS-I equations. It is to be noted that the Eq. (31) has necessarily assumed that $k_{\|} \neq 0$. The existence of the DS-I equations is satisfied only for a specific set of transformations satisfying Eq. (34). We have once again carried out a numerical exploration in parameter space of these conditions. Figures $3 \mathrm{a}$ and $\mathrm{b}$ present the parametric domain of the existence of the DS-I equations in the three-dimensional parameter space of $\alpha, k_{\mathrm{y}}$ and $\omega$ for $\beta=0$ and 0.1 , respec- tively. Figures $4 \mathrm{a}$ and $\mathrm{b}$ represent the corresponding twodimensional projections in the $\alpha-\omega$ plane, respectively, for different $k_{\mathrm{y}}$ and $k_{\mathrm{z}}$, whereas Figs. 5a and b represent similar projections in the $\alpha-\theta$ plane, with $\theta$ being the angle of propagation with respect to the ambient magnetic field. We note that, analogous to the perpendicular propagation case, for oblique propagation also, an increasing $\beta$ favors the conditions of DS-I equations (e.g. Figs. 3a and b, respectively). But in contrast to the previous case, the allowed range of $\beta$ values is found to be more restricted for an oblique propagation. In fact, there appears to be an upper cutoff in $\beta$ (i.e. $\beta \leq \beta_{\mathrm{c}}$, where $\beta_{\mathrm{c}} \approx 0.17$ ) beyond which the equations cannot be reduced to the DS-I form.

\section{Comparison with the space plasma observations}

Our numerical results presented in the previous sections display the regions in parameter space where the propagation 


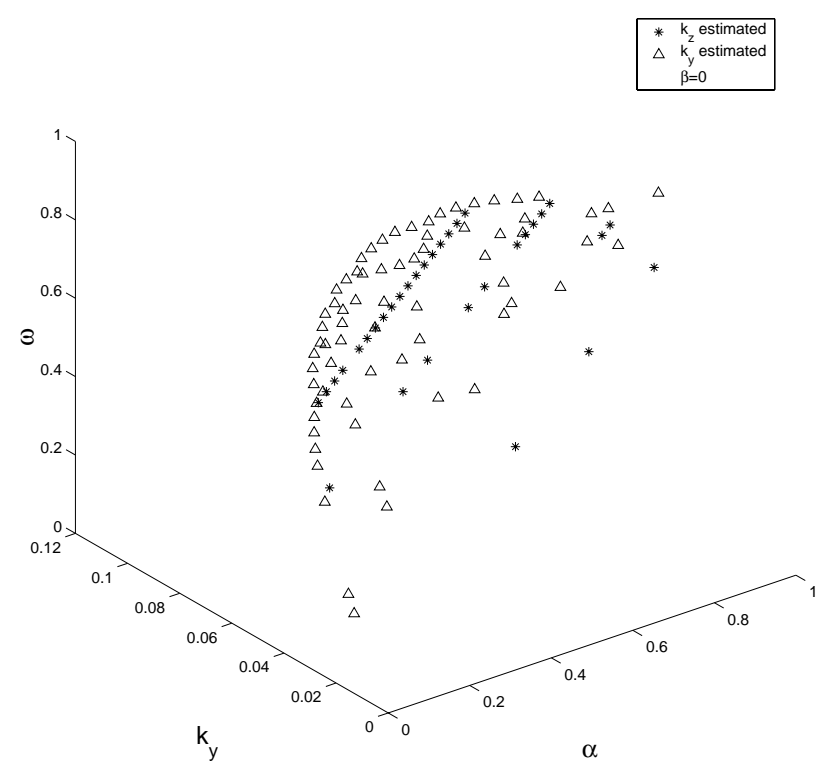

Fig. 3a. Existence domain for DS-I equation for oblique propagation; $\beta=0$.

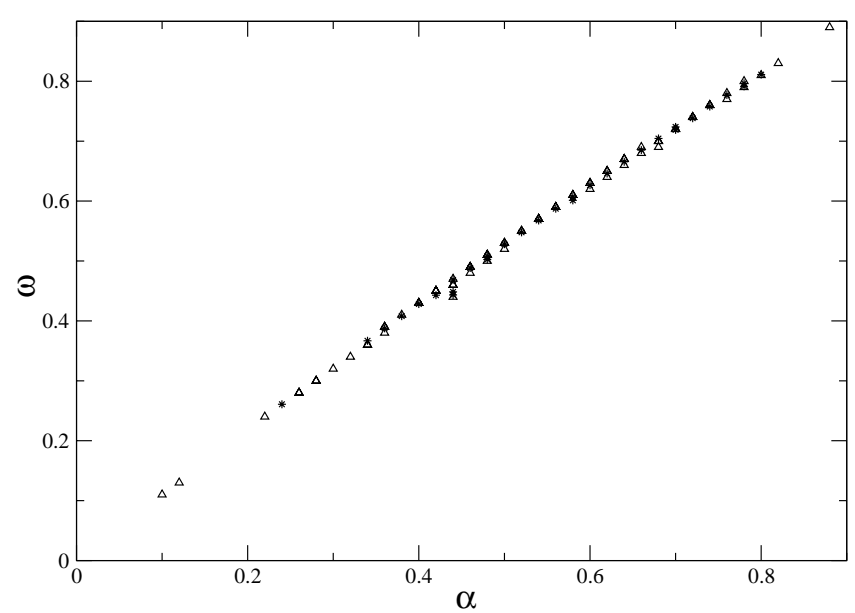

Fig. 4a. $\alpha$ vs. $\omega$ for oblique propagation $\beta=0$.

equation can be represented by the DS-I equations. For the case of perpendicular propagation, where condition (27) needs to be satisfied, the parameter space is represented by $\alpha$ and $\omega$, with $\omega$ being calculated from the dispersion rela-

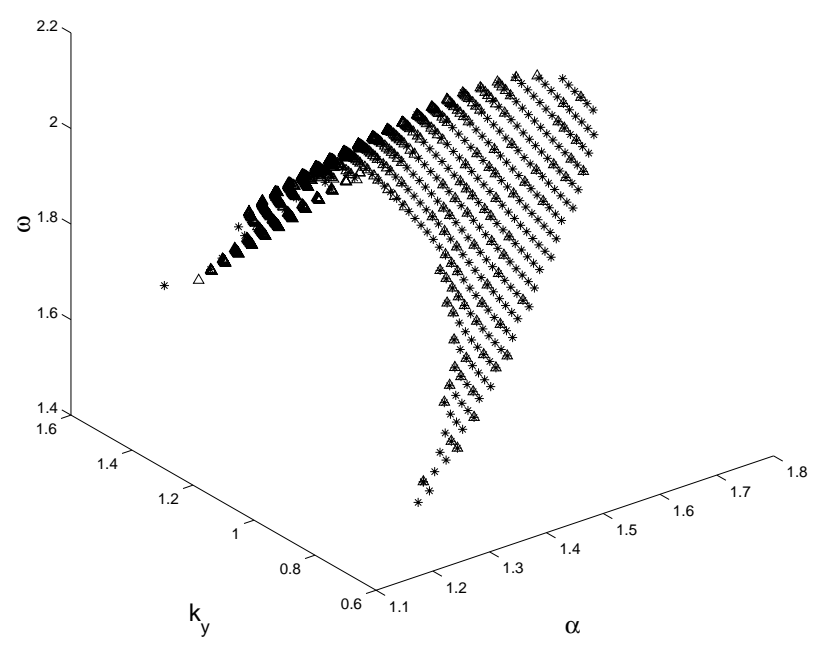

Fig. 3b. Existence domain for DS-I equation for oblique propagation; $\beta=0.1$.

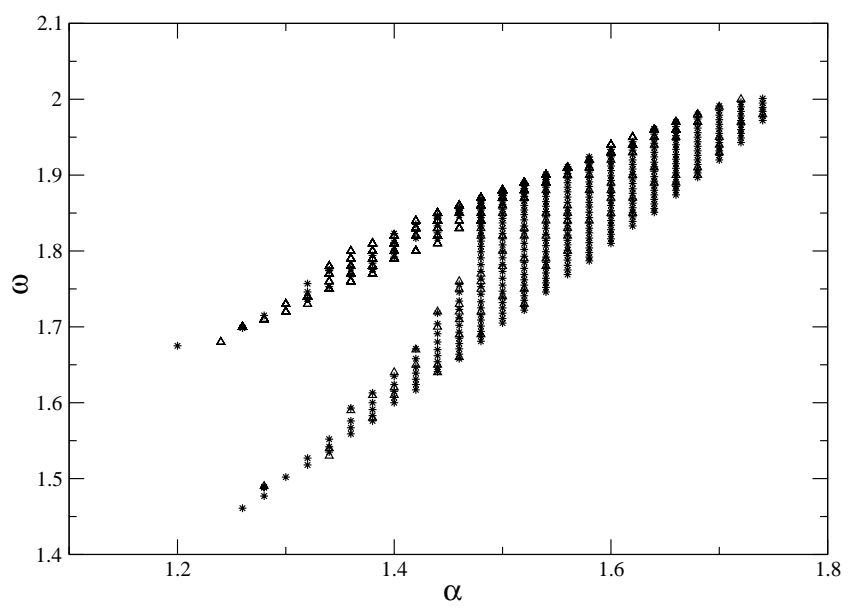

Fig. 4b. $\alpha$ vs. $\omega$ for oblique propagation; $\beta=0.1$.

tion (10). Figure 1a displays the results for cold electrons (i.e. $\beta=0$ ), while Figs. $1 \mathrm{~b}$ and $1 \mathrm{c}$ represent $\beta=0.1$ and $\beta=1$, respectively. We now check to see if these parametric regions include the physical parameter range corresponding

Table 1. Wave frequencies for different $f_{\text {ce }}$

\begin{tabular}{cccc}
\hline $\begin{array}{c}f_{\text {ce }} \\
(\mathrm{kHz})\end{array}$ & $\begin{array}{c}\alpha \\
\text { (normalized) }\end{array}$ & $\begin{array}{c}\omega \\
\text { (normalized) }\end{array}$ & $\begin{array}{c}1 / f_{\omega} \\
(\mathrm{ms})\end{array}$ \\
\hline 2 & 0.2 & 0.3 & 0.33 \\
5 & 0.5 & 0.6 & 0.17 \\
8 & 0.8 & 0.9 & 0.11 \\
\hline
\end{tabular}

Table 2. Width ratios for different $\alpha$

\begin{tabular}{cccc}
\hline$\alpha$ & $k$ & $\omega$ & $\rho$ \\
\hline 0.2 & 0.2 & 0.301 & 0.238 \\
0.5 & 0.35 & 0.629 & 0.635 \\
0.8 & 0.55 & 0.981 & 0.849 \\
\hline
\end{tabular}




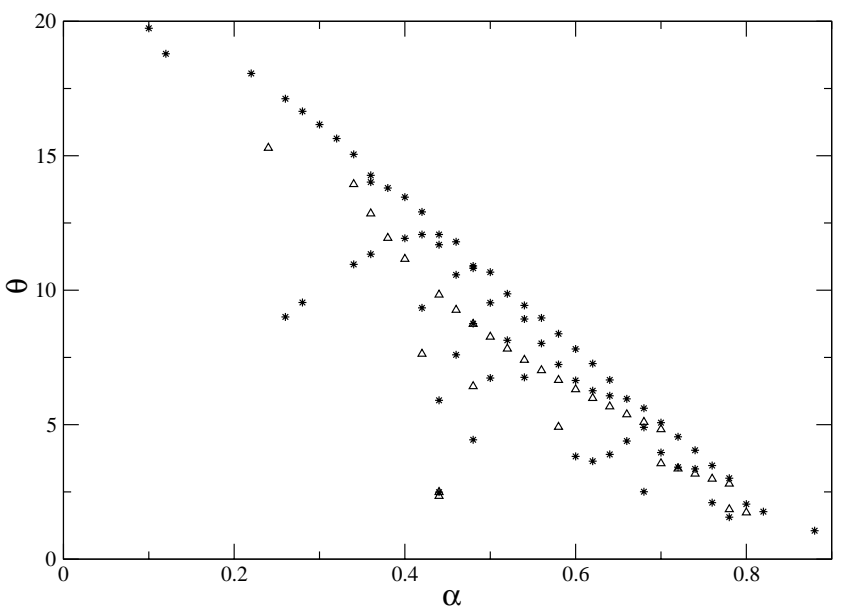

Fig. 5a. $\alpha$ vs. $\theta$ for oblique propagation; $\beta=0$.

to experimental observations. The typical measured parameters in the region of the satellite observations (at a height of 5 to 7 Earth radii) are $T_{\mathrm{i}}=100 \mathrm{eV}, T_{\mathrm{e}}=10 \mathrm{eV}$ (i.e. $\beta=0.1$ ), $n \simeq 1 \mathrm{~cm}^{-3}\left(f_{\mathrm{pe}} \simeq 10 \mathrm{kHz}\right)$ and $f_{\mathrm{ce}} \simeq 5$ to $8 \mathrm{kHz}$ (i.e. $\alpha=0.5$ to 0.8 ) (Tsurutani et al., 1998). We have chosen three values of $f_{\text {ce }}(\alpha)$ and computed the corresponding $\omega$ values from Fig. 1. The result is summarized in Table 1. Since the experimentally observed duration of the nonlinear structures is of the order of $1 \mathrm{~ms}$ or less (Tsurutani et al., 1998), our calculated values of $\omega$ appear to be consistent with the observations. The narrowing of the electronic pulse with increasing $\alpha$ observed in Table 1 is maintained for other $\beta$ values, as indicated in Figs. 1a-c. The same trend continued for arbitrary (oblique) propagation as well which is evident from Figs. 4a and $b$, respectively.

In Fig. 2, we have shown a possible dromion solution for a chosen set of parameters (i.e. $\alpha=0.9, \omega=1.098$, with $\beta$ being 0.1) at time $\mathrm{t}=0$ (Appendix B). For simplicity, we have assumed equal width for both directions. We have compared the shape and size of our solution with that of the experimentally observed structures where the physical conditions are assumed to remain the same (i.e. $f_{\mathrm{pe}}=10 \mathrm{kHz}, T_{\mathrm{i}}=$ $100 \mathrm{eV}, \beta$ being 0.1). According to Franz et al. (1998), the typical parallel scale sizes of the coherent electric field structures corresponding to recent POLAR observations are of the order of 100-1000 m, whereas the calculated half width of the dromion in Fig. 2 is around $166.8 \mathrm{~m}$ (2.5 Debye length), which agrees fairly well with the experimental observations. Regarding the perpendicular scale size, we have calculated the width ratio ( $\rho=L_{\|} / L_{\perp}, L_{\|}\left(L_{\perp}\right)$ being the parallel (perpendicular) scale sizes, respectively) from the following analytic relation derived by Franz et al. (2000)

$\rho \simeq\left(1+r_{\mathrm{L}}^{2}\right)^{-1 / 2}$

where $r_{\mathrm{L}}$ is the normalized electron gyroradius. Setting $r_{\mathrm{L}}=M_{\mathrm{y}} / \alpha$ and using Eq. (18), we have obtained $\rho=1.035$, indicating a near spherical structure (i.e. $L_{\|} \approx L_{\perp}$ ) for our chosen set of parameters. This also confirms the validity

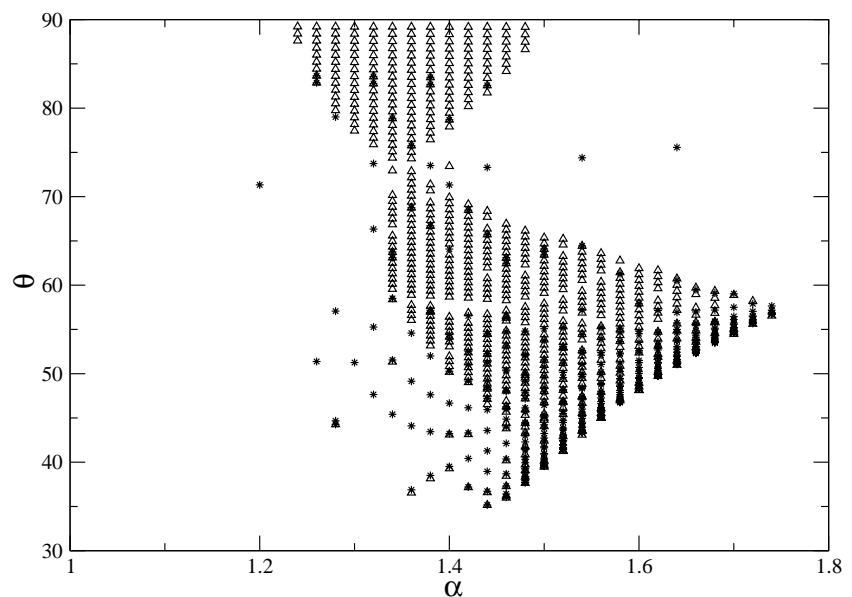

Fig. 5b. $\alpha$ vs. $\theta$ for oblique propagation; $\beta=0.1$.

of our assumption of a spherical dromion in Fig. 2. In order to find out the amplitude ( $a=0.52$ in Fig. 2$)$ we have used Eq. (8) and the corresponding gradient is numerically estimated from Fig. 2. The calculated amplitude is around $0.75 \mathrm{mV} / \mathrm{m}$ while the typical amplitudes of the structures are reported as being of the order of $1 \mathrm{mV} / \mathrm{m}$ (Franz et al., 2000). Hence, our calculated estimation seems to agree quite satisfactorily with the recent spacecraft observations in the PCBL regions. The POLAR observations have further indicated that the shapes of the two dimensional structures depend crucially on $\alpha$ (Franz et al., 2000), being nearly spherical for $\alpha>1$ while becoming more oblate $\left(L_{\|}<L_{\perp}\right)$ with decreasing $\alpha$ (i.e. $\alpha<1$ ). In Table 2 we have calculated $\rho$ by using Eq. (35) for three different values of $\alpha$ and $k$ which satisfy the condition for DS-I equations (Fig. 1b). The decreasing value of $\rho$ with decreasing $\alpha$ indicates an expected oblate shape for the possible solutions. Our theoretical model thus remain consistent with present observational data.

The question of the direction of propagation of the nonlinear pulses has not been satisfactorily established yet. Earlier studies by Gurnet et al. (1976) and Onsager et al. (1993) indicated the possibilities of perpendicular propagation but later observations (Kojima et al., 1997) found inconsistencies with this conclusion. In fact, most of the current experimental observations (Franz et al., 1998; Ergun et al., 1998) and some of the past analytic studies (Goldman et al., 1999; Muschietti et al., 1999) emphasize that propagation of these nonlinear pulses is near parallel. Our theoretical analysis for oblique propagation provides a framework for looking at such a possibility. From Fig. 5a we see that dromion solutions with near parallel propagation $\left(\theta<10^{\circ}\right)$ are also possible in the region of $\alpha \sim 0.5$ for very low electron temperatures. With increasing $\alpha(\alpha \geq 0.7)$, the propagation angle seems to reduce further $\left(\theta \leq 5^{\circ}\right.$; Fig. 5a), strengthening the possibilities of such a near parallel propagation. However, in the absence of more definitive data on the direction of propagation of the pulses, it is not very meaningful to define any realistic quantitative bounds on the existence domain of these structures 
on the basis of our model.

\section{Conclusion}

In the present work, we have shown that the nonlinear evolution of two-dimensional electron acoustic waves can be modelled by the Davey-Stewartson-I equations. These equations admit dromion solutions which are generalizations of soliton solutions to two dimensions and exhibit exponential spatial decay in both directions. Since experimentally observed potential structures in the PCBL region appear to have a distinctly two-dimensional structure, it would be meaningful to characterize them in terms of such two-dimensional nonlinear entities. The basis for the present description rests on the primary assumption of the excitation and sustenance of electron acoustic waves in the PCBL region. Such a proposition for one-dimensional wave has been investigated by many authors (Buti, 1980; Dubuloz et al., 1991a,b; Singh et al., 2001). Using high resolution data of FAST, Pottelette et al. (2001) presented evidence for the presence of nonlinear electron acoustic waves in the auroral kilometric radiation source region. Experimental evidence pointing to the existence of energetic ions $\left(T_{i}>T_{e}\right)$ in the PCBL region (Tsurutani et al., 1998) further supports the assumption of sustaining electron acoustic wave in that region (Buti, 1980; Omura et al., 1994). In fact, there is considerable past work devoted to the question of the generation mechanism of this wave (Bharuthram and Shukla, 1988; Singh and Lakhina, 2001) and its stability (Lashmore-Davies and Martin, 1973). Our present work can be viewed as a natural extension of these investigations towards the description of a nonlinear saturated state of the wave in two spatial dimensions. One possible saturation mechanism of such two-dimensional structures may be the modulational instability as observed experimentally by Pottelette et al. (1999). This can be conjectured further in the context of the stability analysis presented by Nishinari et al. (1994) for the ion acoustic wave. It should be mentioned that the physical saturation mechanisms for a two-dimensional electron acoustic wave would be quite analogous and similar in nature to what has been proposed for the case of the ion acoustic wave (Nishinari et al., 1994). In other words, a twodimensional localization can arise due to the simultaneous onset of a self-focusing instability in the transverse direction and a modulational instability in the longitudinal direction. The evolution and saturation of such an unstable fluctuation into a dromion state can happen if the DS-I condition is satisfied. In our present work we have used this condition to define a parametric space relating the plasma equilibrium quantities and wave propagation characteristics. We have delineated this parameter region by a detailed numerical investigation of the constraint conditions both for the special case of perpendicular propagation and for the general case of oblique propagation. Comparisons with available experimental data show that dromion solutions can exist both for perpendicular propagation, as well as for nearly parallel propagation of the electron acoustic mode. The shape and size of our analytical solution was found to agree well with those observed in POLAR spacecraft measurements. This further strengthens the possibilities of the physical existence of dromions in the auroral plasmas. However, for a more quantitative understanding of the shape and finer features of the observed structure, one needs to carry out a detailed numerical investigation of the evolution equation with appropriate initial conditions. Such an investigation is in progress and will be reported elsewhere. Our present motivation was to provide an alternate paradigmatic model for the observed structures and to demonstrate the basis of its applicability. Further, it should also be pointed out that there can be competing processes in the PCBL region involving other low frequency waves, such as the lower hybrid waves (Dubuloz et al., 1991a). A nonlinear analysis of such waves, along the present lines, to examine two-dimensional solutions would be very worthwhile and to the best of our knowledge has not been accomplished to date. Thus, dromions offer a rich and new paradigm for understanding plasma wave phenomena in the auroral plasma and need to be investigated more intensely.

Appendix A Coefficients for the equations

$$
\begin{aligned}
& a_{1}=\frac{\omega}{\omega^{2}-\alpha^{2} b_{\mathrm{z}}^{2}}, \quad a_{2}=\frac{\alpha b_{\mathrm{z}}}{\omega^{2}-\alpha^{2} b_{\mathrm{z}}^{2}}, \quad a_{3}=\frac{\omega^{2}+\alpha^{2} b_{\mathrm{z}}^{2}}{\omega^{2}-\alpha^{2} b_{\mathrm{z}}^{2}}, \quad a_{12}^{2}=a_{1}^{2}+a_{2}^{2}, \quad a_{4}=a_{1}^{2}+3 a_{2}^{2} \\
& K_{1}=\frac{1}{1+|k|^{2}}, \quad K_{2}=3 \beta+K_{1}, \quad K_{3}=3 \beta-K_{1}^{2}, \quad K_{4}=K_{2}+K_{3}, \quad K_{5}=3 \beta+K_{1}^{2} \\
& c_{1}=a_{1} k_{\perp}^{2}+\frac{k_{\|}^{2}}{\omega}, \quad c_{2}=a_{1} k_{\perp} K_{2}, \quad c_{3}=a_{12}^{2} k_{\perp}^{2}+\frac{k_{\|}^{2}}{\omega^{2}}, \quad c_{4}=a_{1} a_{4} k_{\perp}^{2}+\frac{k_{\|}^{2}}{\omega^{3}} \\
& a_{1 \omega}=\frac{2 \omega}{4 \omega^{2}-\alpha^{2} b_{\mathrm{z}}^{2}}, \quad a_{2 \omega}=\frac{\alpha b_{\mathrm{z}}}{4 \omega^{2}-\alpha^{2} b_{\mathrm{z}}^{2}}, \quad a_{3 \omega}=\frac{\omega^{2}+2 \alpha^{2} b_{\mathrm{z}}^{2}}{\omega^{2}-\alpha^{2} b_{\mathrm{z}}^{2}} \\
& a_{12 \omega}^{2}=a_{1} a_{1 \omega}+a_{2} a_{2 \omega}, \quad a_{12}^{\prime}=2 \omega a_{1}+\alpha b_{\mathrm{z}} a_{2}, \quad a_{21}^{\prime}=\alpha b_{\mathrm{z}} a_{1}+2 \omega a_{2}, \quad a_{4 \omega}=a_{1 \omega} a_{3 \omega}
\end{aligned}
$$




$$
\begin{aligned}
& \kappa_{1}=\frac{1}{1+4|k|^{2}}, \quad \kappa_{2}=3 \beta+\kappa_{1}, \quad \kappa_{3}=3 \beta-\kappa_{1} K_{1}^{2}, \quad \kappa_{4}=K_{2}+\kappa_{3} \\
& c_{1 \omega}=a_{1 \omega} k_{\perp}^{2}+\frac{k_{\|}^{2}}{2 \omega}, \quad c_{2 \omega}=a_{1 \omega} a_{12}^{\prime} k_{\perp}^{2}+\frac{k_{\|}^{2}}{\omega}, \quad c_{3 \omega}=a_{12 \omega}^{2} k_{\perp}^{2}+\frac{k_{\|}^{2}}{2 \omega^{2}}, \quad c_{4 \omega}=a_{1} a_{4 \omega} k_{\perp}^{2}+\frac{k_{\|}^{2}}{\omega^{2}} \\
& X_{\mathrm{x} \eta}=a_{1} K_{2} M_{\mathrm{y}}+k_{\perp} K_{1}^{2}-\frac{1}{2 k_{\perp}} K 2, \quad X_{\mathrm{x} \zeta}=a_{1} K_{2} M_{\mathrm{z}}+k_{\|} K_{1}^{2}, \\
& X_{\mathrm{y} \eta}=a_{3} K_{2} \frac{M_{\mathrm{y}}}{\omega}+2 k_{\perp} K_{1}^{2}-\frac{1}{k_{\perp}} K 2, \quad X_{\mathrm{y} \zeta}=a_{3} K_{2} \frac{M_{\mathrm{z}}}{\omega}+2 k_{\|} K_{1}^{2}, \\
& X_{\mathrm{z \eta}}=K_{2} \frac{M_{\mathrm{y}}}{\omega}+2 k_{\perp} K_{1}^{2}, \quad X_{\mathrm{z \zeta}}=K_{2} \frac{M_{\mathrm{z}}}{\omega}+2 k_{\|} K_{1}^{2}-\frac{1}{k_{\|}} K 2 \\
& S_{\mathrm{x}}=2 a_{2 \omega} \kappa_{2} S_{\mathrm{n}}+f_{\mathrm{x}}, \quad S_{\mathrm{y}}=2 a_{1 \omega} \kappa_{2} S_{\mathrm{n}}+f_{\mathrm{y}}, \quad S_{\mathrm{z}}=\frac{\kappa_{2}}{\omega} S_{\mathrm{n}}+f_{\mathrm{z}}, \quad S_{\mathrm{n}}=\frac{\omega+f_{\mathrm{yz}}}{\omega-2 c_{1 \omega} \kappa_{2}} \\
& f_{\mathrm{x}}=\frac{1}{2} a_{1 \omega} a_{21}^{\prime} K_{2}+a_{2 \omega} \kappa_{3}, \quad f_{\mathrm{y}}=\frac{1}{2} a_{1 \omega} a_{12}^{\prime} K_{2}+a_{1 \omega} \kappa_{3}, \quad f_{\mathrm{z}}=\frac{1}{2 \omega}\left(K_{2}+\kappa_{3}\right)=\frac{\kappa_{4}}{2 \omega}, \\
& f_{\mathrm{yz}}=k_{\perp}^{2} f_{\mathrm{y}}+k_{\|}^{2} f_{\mathrm{z}}=\frac{1}{2} c_{2 \omega} K_{2}+c_{1 \omega} \kappa_{3}, \quad f_{\mathrm{k}}=c_{4 \omega} K_{2}^{2}+2 c_{3 \omega} K_{2}\left(2 \kappa_{2} S_{\mathrm{n}}+\kappa_{3}\right)+\kappa_{4} S_{\mathrm{n}}-\kappa_{1} K_{1}^{2} \\
& p_{\eta \eta}=0, \quad p_{\eta \zeta}=2 M_{\mathrm{y}} K_{2} \frac{k_{\|}}{\omega}\left(1+a_{2}^{2} k_{\perp}^{2} K_{2}\right), \quad p_{\zeta \zeta}=2 M_{\mathrm{z}} K_{2} \frac{k_{\|}}{\omega}\left(1+a_{2}^{2} k_{\perp}^{2} K_{2}\right)+K_{4} \\
& q_{\eta \eta}=2 \frac{k_{\|}}{\omega}\left(a_{2} k_{\perp} K_{2}\right)^{2} M_{\mathrm{y}}^{2}, \quad q_{\eta \zeta}=4 \frac{k_{\|}}{\omega}\left(a_{2} k_{\perp} K_{2}\right)^{2} M_{\mathrm{y}} M_{\mathrm{z}}+M_{\mathrm{y}} K_{4}, \quad q_{\zeta \zeta}=2 \frac{k_{\|}}{\omega}\left[\left(a_{2} k_{\perp} K_{2}\right)^{2} M_{\mathrm{z}}^{2}+(3 \beta+1) K_{2}\right]+K_{4} M_{\mathrm{z}} \\
& \mu_{02}=\frac{c_{1} K_{4}}{1+c_{3} K_{2}}, \quad \mu_{11}=\frac{c_{1} f_{\mathrm{k}}}{1+c_{3} K_{2}}-2 c_{2} k_{\perp}, \quad \mu_{\xi \xi}=\frac{a_{1} K_{2}-c_{1} K_{1}^{2}}{1+c_{3} K_{2}}, \\
& \mu_{\eta \eta}=\frac{1}{1+c_{3} K_{2}}\left[c_{4} K_{2} M_{\mathrm{y}}^{2}+2 k_{\perp} M_{\mathrm{y}}\left(c_{3} K_{1}^{2}-a_{12}^{2} K_{2}\right)+4 k_{\perp}^{2} K_{1}^{2}\left(c_{1} K_{1}-a_{1}\right)+\left(a_{1} K_{2}-c_{1} K_{1}^{2}\right)\right] \\
& \mu_{\eta \zeta}=\frac{2}{1+c_{3} K_{2}}\left[c_{4} K_{2} M_{\mathrm{y}} M_{\mathrm{z}}+c_{3} K_{1}^{2}\left(k_{\perp} M_{\mathrm{z}}+k_{\|} M_{\mathrm{y}}\right)-K_{2}\left(a_{12}^{2} k_{\perp} M_{\mathrm{z}}+\frac{1}{\omega^{2}} k_{\|} M_{\mathrm{y}}\right)-2 k_{\perp} k_{\|} K_{1}^{2}\left(a_{1}+\frac{1}{\omega}-2 c_{1} K_{1}\right)\right] \\
& \mu_{\zeta \zeta}=\frac{1}{1+c_{3} K_{2}}\left[c_{4} K_{2} M_{\mathrm{z}}^{2}+2 k_{\|} M_{\mathrm{z}}\left(c_{3} K_{1}^{2}-\frac{K_{2}}{\omega^{2}}\right)+4 k_{\|}^{2} K_{1}^{2}\left(c_{1} K_{1}-\frac{1}{\omega}\right)+\left(\frac{K_{2}}{\omega}-c_{1} K_{1}^{2}\right)\right] \\
& e_{1}=\frac{M_{\mathrm{y}}^{2}}{3 \beta+1-M_{\mathrm{z}}^{2}}, \quad e_{2}=\frac{2 M_{\mathrm{y}} M_{\mathrm{z}}}{3 \beta+1-M_{\mathrm{z}}^{2}}, \quad e_{3}=\frac{R_{\eta \eta}+e_{1} R_{\zeta \zeta}}{R_{\eta \zeta}+e_{2} R_{\zeta \zeta}} \\
& R_{\alpha \beta}=\frac{r_{\alpha \beta}}{1+3 \beta-M_{\mathrm{z}}^{2}} \\
& \hat{\boldsymbol{M}}=\left(\begin{array}{ccc}
\mu_{\eta \eta} & \mu_{\eta \zeta} & \mu_{\zeta \zeta} \\
R_{\eta \eta} & R_{\eta \zeta} & R_{\zeta \zeta} \\
-e_{1} & -e_{2} & 1
\end{array}\right) \quad ; \quad \hat{\boldsymbol{T}}=\left(\begin{array}{c}
2 p_{\mathrm{y}} p_{\mathrm{z}} \\
p_{\mathrm{y}} q_{\mathrm{z}}+p_{\mathrm{z}} q_{\mathrm{y}} \\
q_{\mathrm{y}} q_{\mathrm{z}}
\end{array}\right)
\end{aligned}
$$

Provided det $|\hat{\boldsymbol{M}}|=0$

$\frac{q_{\mathrm{y}}}{p_{\mathrm{y}}}=E \frac{p_{\mathrm{z}}}{q_{\mathrm{z}}} ; \frac{q_{\mathrm{z}}}{p_{\mathrm{z}}}+\frac{q_{\mathrm{y}}}{p_{\mathrm{y}}}=-2 e_{3}$

Assuming $\frac{p_{\mathrm{z}}}{q_{\mathrm{z}}}=t$,

$$
\begin{aligned}
& t=\frac{-e_{3} \pm \sqrt{e_{3}^{2}-E}}{E} \\
& l_{\mathrm{y}}=\frac{2 \mu_{11}}{K_{4} \mu_{02}} \frac{d_{\zeta}}{d_{\eta}} M_{\mathrm{y}}^{2}, \quad l_{\mathrm{z}}=\frac{2 \mu_{11}}{K_{4} \mu_{02}}(3 \beta+1)
\end{aligned}
$$




\section{Appendix B Hirota bilinear method}

We briefly outline the Hirota bilinear method here and apply it to obtain an exact analytic one-dromion solution for the perpendicular propagation case. We first rescale the dependent and independent variables as

$\eta=\sqrt{\frac{2 d_{\eta}}{\mu_{11}}} Y ; \quad \zeta=\sqrt{\frac{2 d_{\zeta}}{\mu_{11}}} Z ; \quad \tau=\frac{1}{\mu_{11}} T$,

$\frac{\mu_{02}}{\mu_{11}} Q=q ; A=a$.

Using Eq. (B1), Eqs. (25) and (26) transform to

$\iota a_{\mathrm{T}}+\frac{1}{2}\left(a_{\mathrm{YY}}+a_{\mathrm{ZZ}}\right)-\left(q+|a|^{2}\right) a=0$,

$l_{\mathrm{y}} q_{\mathrm{YY}}-l_{\mathrm{Z}} q_{\mathrm{ZZ}}-2|a|_{\mathrm{ZZ}}^{2}=0$,

where $l_{\mathrm{y}}$ and $l_{\mathrm{z}}$ are given in Appendix A.

Putting the usual substitutions (Radha and Lakshmanan, 1997a)

$a=\frac{g}{f} ; \quad q=-2 \partial_{\mathrm{z}}^{2} \ln f$,

with the assumption $l_{\mathrm{y}}, l_{\mathrm{z}} \approx 1$ and rotating the coordinate axis by $45^{\circ}$, Eqs. (B2) and (B3) reduce to the bilinear form as

$\left[{ }_{l} D_{\mathrm{T}}+\frac{1}{2}\left(D_{\mathrm{Y}}^{2}+D_{\mathrm{Z}}^{2}\right)\right] g \cdot f=0$,

$D_{\mathrm{Y}} D_{\mathrm{Z}} f \cdot f=-2 g \cdot g^{*}$,

where $D_{\mathrm{Y}, \mathrm{Z}}$ are the Hirota bilinear operators. Expanding the new variables as

$g=\epsilon g^{(1)}+\epsilon^{3} g^{(3)}+\ldots$,

$f=1+\epsilon^{2} f^{(2)}+\epsilon^{4} f^{(4)}+\ldots$,

with $\epsilon$ being a small quantity, and equating for different orders we obtain

$\epsilon: l g_{\mathrm{T}}^{(1)}+\frac{1}{2}\left(g_{\mathrm{YY}}^{(1)}+g_{\mathrm{ZZ}}^{(1)}\right)=0$,

$\epsilon^{2}: f_{\mathrm{YZ}}^{(2)}=g^{(1)} g^{*(1),}$

$\epsilon^{3}: l g_{\mathrm{T}}^{(3)}+\frac{1}{2}\left(g_{\mathrm{YY}}^{(3)}+g_{\mathrm{ZZ}}^{(3)}\right)=$

$-\left[{ }_{l} D_{\mathrm{T}}+\frac{1}{2}\left(D_{\mathrm{Y}}^{2}+D_{\mathrm{Z}}^{2}\right)\right] g^{(1)} \cdot f^{(2)}$,

$\epsilon^{4}: f_{\mathrm{YZ}}^{(4)}+\frac{1}{2} D_{\mathrm{Y}} D_{\mathrm{Z}}\left(f^{(2)} \cdot f^{(2)}\right)=$

$\left(g^{(1)} \cdot g^{*(3)}+g^{(3)} \cdot g^{*(1)}\right)$

and so on. Solving Eq. (B7) we obtain

$g^{(1)}=\sum_{j=1}^{N} \exp \chi_{\mathrm{j}}, \quad \chi_{\mathrm{j}}=p_{\mathrm{j}} Y+s_{\mathrm{j}} Z-\imath \omega T+b_{\mathrm{j}}$,

where $p_{\mathrm{j}} s_{\mathrm{j}}$ and $b_{\mathrm{j}}$ are complex constants satisfying the condition

$\frac{1}{2}\left(p_{\mathrm{j}}^{2}+s_{\mathrm{j}}^{2}\right)-\omega=0$.
To construct a one-dromion solution, we set $N=1$. Substituting Eq. (B11) in Eq. (B8) we obtain

$f^{(2)}=\exp \left(\chi_{1}+\chi_{1}^{*}+2 \psi\right), \exp (2 \psi)=\frac{1}{4 p_{1 R} s_{1 R}}$,

with $p_{1 R}, s_{1 R}$ being the real part of $p_{1}, s_{1}$, respectively. Using $g^{(1)}, f^{(2)}$ from Eqs. (B7) and (B8) into Eqs. (B9) and (B10), one can show that $g^{(\mathrm{j})}=0$ for $j \geq 3$ and $f^{(j)}=0$ for $j \geq 4$, respectively. Now using Eqs. (11) and (13) in Eq. (B4), the physical quantities $a$ and $q$ will assume the following form

$a=\sqrt{p_{1 \mathrm{R}} s_{1 \mathrm{R}}} \operatorname{sech}\left(\chi_{1 \mathrm{R}}+\psi\right) \exp \left(l \chi_{1 \mathrm{I}}\right)$,

$q=-2 s_{1 \mathrm{R}}^{2} \operatorname{sech}^{2}\left(\chi_{1 \mathrm{R}}+\psi\right)$.

To obtain an analytic form for a dromion solution, we use the ansatz (Radha and Lakshmanan, 1997a)

$$
\begin{gathered}
f=1+\exp \left(\chi_{1}+\chi_{1}^{*}\right)+\exp \left(\chi_{2}+\chi_{2}^{*}\right) \\
+\exp \left(\chi_{1}+\chi_{1}^{*}+\chi_{2}+\chi_{2}^{*}\right),
\end{gathered}
$$

where

$$
\begin{aligned}
& \chi_{1}=p_{1} Y+\imath \frac{p_{1}^{2}}{2} T+b_{1} \\
& \chi_{2}=s_{1} Y+\imath \frac{s_{1}^{2}}{2} T+b_{2} .
\end{aligned}
$$

Substituting Eq. (B16) with Eq. (B17) and putting it into Eq. (B8), we obtain

$g=\gamma \exp \left(\chi_{1}+\chi_{2}\right) ;|\gamma|^{2}=4 p_{1 \mathrm{R}} s_{1 \mathrm{R}}$

For simplicity, we have assumed $p_{1}=s_{1}=p$. For $\mathrm{T}=0$ the dromion solution for $a$ is obtained as

$a(Y, Z, T=0)$

$$
=\frac{2 p \exp [p(Y+Z)]}{1+\exp (2 p Y)+\exp (2 p Z)+\exp [2 p(Y+Z)]} .
$$

Numerically we have obtained $l_{\mathrm{y}} \approx l_{\mathrm{z}} \approx 0.825$ for $\alpha=0.9$ and $k_{\mathrm{y}}=0.6234(\omega=1.098)$. Calculating $p$ from Eq. (B12) and putting it into Eq. (B19), we obtain the solution as presented in Fig. 2.

Acknowledgements. One of the authors, S. S. Ghosh, would like to thank the Council of Scientific and Industrial Research, India, for its financial support.

\section{References}

Ablowitz, M. J. and Segur, H.: On the evolution of packets of water waves, J. Fluid Mech., 92, 691-715, 1979.

Baumjohann, W., Paschmann, G., and Cattell, C. A.: Average plasma properties in the central plasma sheet, J. Geophys. Res., 94, 6597-6606, 1989.

Besse, C. and Bruneau, C.: Mathematical Models \& Methods in Applied Sciences, 8, 1363-1386, 1998.

Bharuthram, R. and Shukla, P. K.: Nonlinear properties of electron acoustic wave turbulence in the geomagnetic tail, Astrophys. Space Sc., 149, 127-149, 1988. 
Boiti, M., Leon J. J. P., Martina, L., and Pempinelli, F.: Scattering of localized solitons in the plane, Phys. Lett., 132A, 432-439, 1989.

Buti, B.: Nonlinear electron acoustic waves in a multi-species plasma, J. Plasma Phys., 24, 169-180, 1980.

Buti, B., Mohan, M., and Shukla, P. K.: Exact electron-acoustic solitary waves, J. Plasma Phys., 23, 341-347, 1979.

Davey, A. and Stewartson, K.: On three-dimensional packets of surface waves, Proc. R. Soc. Lond., 338A, 101-110, 1974.

Djordjevic, V. D. and Redekopp, L. G.: On two-dimensional packets of capillary-gravity waves, J. Fluid Mech., 79, 703-714, 1977.

Dubuloz, N., Pottelette, R., Malingre, M., and Treumann, R. A.: Generation of broadband electrostatic noise by electron acoustic soliton, Geophys. Res. Lett., 155-158, 1991a.

Dubuloz, N., Pottelette, R., Malingre, M., Holmgren, G., and Lindqvist, P. A.: Detailed analysis of broadband electrostatic noise in the dayside auroral zone, J. Geophys. Res., 96, 35653579, $1991 b$.

Ergun, R. E., Carlson, C. W., McFadden, J. P., Mozer, F. S., Delory, G. T., Peria, W., Chaston, C. C., Temerin, M., Roth, I., Muschietti, L., Elphic, R., Strangeway, R., Pfaff, R., Cattell, C. A., Klumpar, D., Shelley, E., Peterson, W., Moebius E., and Kistler, L.: FAST satellite observations of large-amplitude solitary structures, Geophys. Res. Lett., 25, 2041-2044, 1998.

Fokas, A. S. and Santini, P. M.: Coherent structures in multidimensions, Phys. Rev. Lett., 63, 1329-1333, 1989.

Fokas, A. S. and Santini, P. M.: Dromions and a boundary value problem for the Davey-Stewartson I equation, Physica D, 44, 99130, 1990.

Franz, J. R., Kintner, P. M., and Pickett, J. S.: POLAR observations of coherent electric field, Geophys. Res. Lett., 25, 1277-1280, 1998.

Franz, J. R., Kintner, P. M., Seyler, C. E., Pickett, J. S., and Scudder, C. E.: On the perpendicular scale of electron phase-space, Geophys. Res. Lett., 27, 169-172, 2000.

Gage, K. S. and Reid, G. C.: Solar variability and the secular variation in the tropical tropopause, Geophys. Res. Lett., 8, 187-190, 1981.

Ghosh, S. S., Ghosh, K. K., and Iyengar Sekar A. N.: Large Mach number ion acoustic rarefactive solitary waves for a two electron temperature warm ion plasma, Phys. of Plasmas 3, 3939-3946, (1996).

Goldman, M. V., Oppenheim, M. M., and Newman, D. L.: Nonlinear two strean instabilities as an explanation for auroral bipolar wave structures, Geophys. Res. Lett., 26, 1821-1824, 1999.

Guil, F. and Manas, M.: The Dirac equation and integrable systems of KP type, J. Phys. A: Math. Gen., 29, 641-665, 1996.

Gurnet, D. A., Frank, L. A., and Lepping, R. P.: Plasma waves in the distant magnetotail J. Geophys. Res., 81, 6059-6071, 1976.

Hietarinta, J.: One-dromion solutions for generic classes of equations, Phys. Lett., 149A, 113-118, 1990.

Hietarinta, J. and Hirota, R.: Multidromion solutions to the DaveyStewartson equation, Phys. Lett. A, 145, 237-244, 1990.

Imai, K.: Dromion and lump solutions of the Ishimori-I equation, Prog. Theo. Phys., 98, 1013-1023, 1997.

Janaki, M. S., Som, B. K., Dasgupta, B., and Gupta, M. R.: K$\mathrm{P}$ Burgers equation for the decay of solitary magnetosonic waves propagating obliquely in a warm collisional plasma, J. Phys. Soc. Jpn., 60, 2977-2984, 1991.

Kadomtsev, B. B. and Petviashvili, V. I.: On stability of solitary waves in weakly dispersive media, Sov. Phys. Dokl., 15, 539-
$541,1970$.

Kojima, H., Matsumoto, H., Miyatake, T., Nagano, I., Fujita, A., Frank, L. A., Mukai, T., Paterson, W. R., Saito, Y., Machida, S., and Anderson, R. R.: Relation between electrostatic solitary waves and hot plasma flow in the plasma sheet boundary layer: GEOTAIL observations, Geophys. Res. Lett., 21, 29192922, 1994.

Kojima, H., Matsumoto, H., Chikuba, S., Horiyama, S., AshourAbdalla, M., and Anderson, R. R.: Geotail waveform observations of broadband/narrowband electrostatic noise in the distant tail, J. Geophys. Res., 102, 14 439-14 455, 1997.

Lashmore-Davies, C. N. and Martin, T. J.: Electrostatic instabilities driven by an electric current perpendicular to a magnetic field, Nuclear Fusion, 13, 193-203, 1973.

Lokenath Debnath, Nonlinear Partial Differential Equations for Scientists and Engineers, 12, 501-527, 1997.

Lou, S-y. and Ruan, H-y.: Revisitation of the localized excitations of the (2+1)- dimensional KdV equation, J. Phys. A Math. Gen., 34, 305-316, 2001.

Manakov, S. V.: Usp. Mat. Nauk, 31, 245, 1976.

Matsumoto, H., Nagano, I., Anderson, R. R., Kojima, H., Hasimoto, K., Tsutsui, M., Okada, T., Kimura, I., Omura, Y., and Okada, M.: Plasma wave obsrvations with GEOTAIL spacecraft, J. Geomag. Geoelectr., 46, 59-95, 1994a.

Matsumoto, H., Kojima, H., Miyatake, T., Omura, Y., Okada, M., Nagano, I., and Tsutsui, M.: Electrostatic solitary wave (ESW) in the magnetotail: BEN wave forms observed by GEOTAIL, Geophys. Res. Lett., 21, 2915-2918, 1994b.

Muschietti, L., Ergun, R. E., Roth, I., and Carlson, W.: Phase space electron holes along magnetic field lines, Geophys. Res. Lett., 26, 1093-1096, 1999.

Nishinari, K., Abe, K., and Satsuma, J.: Multidimensional behavior of an electrostatic ion wave in a magnetized plasma, Phys. Plasmas, 1, 2559-2565, 1994.

Nishinari, K. and Yajima, T.: Numerical studies on stability of Dromion and its collisions, J. Phys. soc. Jpn., 63, 3538-3541, 1994.

Nishinari, K., Yajima, T., and Nakao T.: Time evolution of Gaussian-type initial conditions associated with the DaveyStewartson equations, J. Phys. A: Math. Gen., 29, 4237-4245, 1996.

Omura, Y., Kojima, H., and Matsumoto, H.: Computer simulation of electrostatic solitary waves: a nonlinear model of broadband electrostatic noise, Geophys. Res. Lett., 21, 2923-2926, 1994.

Omura, Y., Matsumoto, H., Miyako, T., and Kojima, H.: Electron beam instabilities as generation mechanismm of electrostatic solitary waves in the magnetotail, J. Geophys. Res., 101, 2685-2697, 1996.

Onsager, T. G., Thomsen, M. F., Elphic, R. C., Gosling, J. T., Anderson, R. R., and Kettman, G.: Electron generation of electrostatic waves in the plasma sheet boundary layer, J. Geophys. Res., 98, 15 509-15 519, 1993.

Pottelette, R., Ergun, R. E., Treumann, R. A., Berthomier, M., Carlson, C. W., McFadden, J. P., and Roth, I.: Modulated electronacoustic waves in auroral density, Geophys. Res. Lett., 26, 26292632, 1999.

Pottelette, R., Treumann, R. A., and Berhomier, M.: Auroral plasma turbulence and the cause of auroral kilometric radiation fine structure, J. Geophys. Res., 106, 8465-8476, 2001.

Radha, R. and Lakshmanan, M.: Dromion like structures in the (2+1)- dimensional breaking soliton equation, Phys. Lett., 197A, 7-12, 1995. 
Radha, R. and Lakshmanan, M.: Localized coherent structures and integrability in a generalized (2+1)-dimensional nonlinear Scrödinger equation, Chaos, Solitons \& Fractals, 8, 17-25, 1997a.

Radha, R. and Lakshmanan, M.: A new class of induced localized coherent structures in the (2+1)-dimensional nonlinear Schrödinger equation, J. Phys. A: Math. Gen. , 30, 3229-3233, 1997b.

Ruan, H-y. and Chen, Y-x.: The study of dromion interactions of (2+1)-dimensional integrable systems, J. Math. Phys., 40, 248 255,1999

Santini, P. M.: Energy exchange of interacting coherent structures in multidimensions, Physica D, 41, 26-54, 1990.

Satsuma, J. and Ablowitz, M. J.: Two-dimensional lumps in nonlinear dispersive systems, J. Math. Phys., 20, 1496-1503, 1979.
Scarf, F. L., Frank, L. A., Ackerson, K. L., and Lepping, R. P.: Plasma wave turbulence at distant crossings of the plasma sheet boundaries and the neutral sheet, Geophys. Res. Lett., 1, 189192,1974

Singh, S. V. and Lakhina, G. S.: Generation of electron-acoustic waves in the magnetosphere, Planet. Space Sc., 49, 107-114, 2001.

Singh, S. V., Reddy, R. V., and Lakhina, G. S.: Broadband electrostatic noise due to nonlinear electron-acoustic waves, Adv. Space Res., 28, 1643-1648, 2001.

Tsurutani, B. T., Ho, C. M., Lakhina, G. S., Buti, B., Arballo, J. K., Picket, J. S., and Gurnett, D. A.: Plasma waves in the dayside polar cap boundary layer: bipolar and monopolar electric pulses and whistler mode waves, Geophys. Res. Lett., 25, 4117-4120, 1998. 